\title{
Research on multifunctional integrated intelligent stair-climbing chair based on ergonomics
}

\author{
Man Zhang, Jingde Huang*, and Chenbo Jiang \\ Zhuhai College of Science and Technology, Zhuhai China
}

\begin{abstract}
In view of the practical problems faced by the group with travel inconvenience, the wheelchairs in today's Chinese market can't meet the needs of society. Taking the integrated function chair as the research object, this paper puts forward the whole structure of the self-help ladder climbing intelligent chair. The chair is designed on the basis of analyzing the working principle of the main and the auxiliary structure. Based on Ergonomics, the composition and driving mode of each core module and auxiliary mechanism are developed, and the three-dimensional model of self-help ladder climbing intelligent chair is established by using UG. Selfhelp intelligent stair-climbing chair is not only conductive to improving the quality of life of special people and easing their family burden, but also has positive significance to the healthy and sustainable development of wheelchair industry.
\end{abstract}

Keywords: Intelligent stair-climbing chair; Multifunctional; Ergonomics; Finite element analysis.

\section{The structure of the self-help ladder smart chair}

At present, electric stair climbers are mainly divided into two categories: load stair climbers and manned stair climbers. The load-carrying stair climber is often used in the field of logistics handling, which can help people to easily move heavy objects up and down the stairs. The manned stair climber is suitable for the disabled and the elderly who need to go up and down stairs, so that it can pass through the stairs of buildings without suitable stairway equipment ${ }^{[1]}$. At present, the stair-climbing wheelchairs in the domestic market can be divided into three common types according to the movement mode: crawler type, star wheel type and leg type ${ }^{[2]}$. However, due to the large weight and volume of crawler wheelchair, walking on flat ground consumes a lot of energy, so its endurance is weak. The star-wheeled stair-climbing wheelchair is small in size and flexible in movement, but it can't be used on flat ground. The difficulty of leg type is that it is difficult to control and develop, and the technology is difficult to master, so it is not suitable for China's large market. The traditional step-by-step stair climbing robot has the problems of cumbersome movements and low efficiency ${ }^{[3]}$.

\footnotetext{
* Corresponding author: jdh925@jluzh.edu.cn
} 
Compared with foreign products and technology that have been developed for several years, domestic wheelchairs have a single form, mostly wheelchairs that can only assist walking, and cannot achieve functions such as automatic ladders and assisting the elderly to get up, and they still need help from others while using the wheelchair. Life is inconvenient. With the development of sensors and artificial intelligence technology, the structural design of the chair itself will inevitably move forward to meet the development direction of the wheelchair market. In order to reduce the quality of the wheelchair and improve its reliability level, mechanization, integration and intelligence have become wheelchairs.

Faced with the independent life problems and psychological dilemmas of special people, the self-help ladder-climbing smart chair must not only have the functions of traditional wheelchairs, but also need to focus on solving the current industry's urgently needed ladder balance function and auxiliary standing function ${ }^{[4]}$. In engineering practice, it is necessary to refer to human body data according to functions when designing the mechanism. Therefore, we first optimize the initial data based on ergonomics knowledge, and then use the structural parameters that meet the needs of users to build a three-dimensional model using UG software. The core functional structure is mainly composed of the upper and lower ladder structure, auxiliary standing mechanism, crawler and other structures. The overall functional structure is shown in Figure 1.

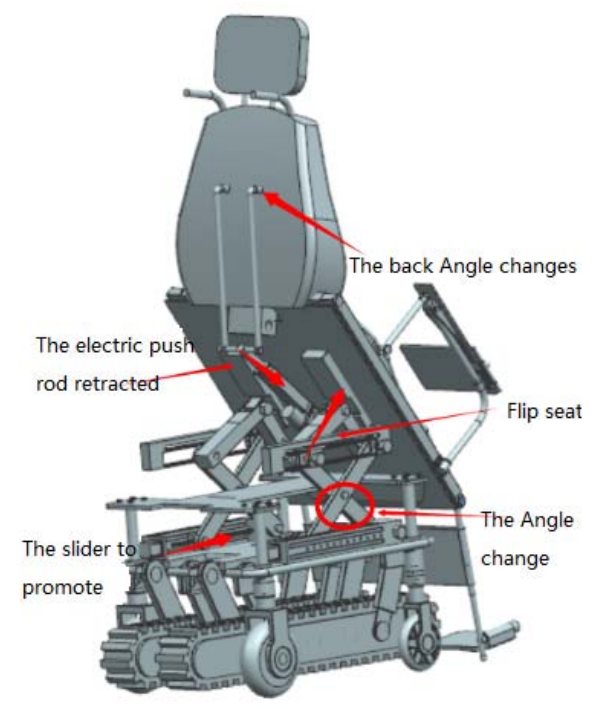

Fig. 1. Structure diagram of self-assisted ladder climbing intelligent chair.

Intelligent self-energizing ladder back chair in an integrated crawler-focused, using the track where the disc is placed, supplementary motor principle four bodies, synthetic movable crawler. Bidirectional motion drive mechanism, the track before and after each set of a drive motor. Six intermediate track using the auxiliary drive gear and the fixed, long straight rod which supplementary intermediate position, i.e., the gear support rods, the shape of the fixation rod. Overall movement mechanism utilizing the principles of the four motion, by the motion of the four-bar mechanism, the vertical ladder when arriving at the position of the track needed to achieve the vertical movement of the ladder track, while adjusting the center of gravity position and movement posture.

Future research should first move towards practical application, which must integrate the application of intelligent technology, optimize control algorithm, enhance automatic planning and sensor-based intelligence. Second, the lightweight of the structure can greatly 
reduce the weight of the internal drive structure and other stress structures, giving users more superior control experience and more stable endurance. The third is to realize speech recognition, as an important part of pattern recognition, its ultimate goal is to realize the natural language communication between man and machine. Speech recognition technology includes feature extraction, pattern matching criteria and model training techniques.

\section{Main structure design}

The most direct contact between the self-service ladder smart chair and the user is the backrest and the seat. Therefore, a comfortable seat and backrest have the most direct impact on the user's use. According to the national standard wheelchair indicators and national stair standards, the width of the backrest is $356 \sim 418 \mathrm{~mm}$, which is basically consistent with the curvature of the human spine, and the backrest Angle is maintained at $100^{\circ} \sim 105^{\circ}$.

\subsection{Tracked chassis design}

This system uses crawlers as the chassis structure of the upper and lower ladders. In the traditional crawler structure, the crawlers are mostly used for the back and the bottom plate. The advantage of the back crawler is that the position of the center of gravity is stable, but the user is not convenient to move; the advantage of the lower-disc crawler is that the human body can see the stairs when going up and down the ladder, and it can be unassisted and has good functionality. Aiming at the above design ideas, integrating the center of gravity of the back-type crawler and the placement of the bottom-disc crawler, we adopted a four-bar mechanism to synthesize a movable crawler, as shown in Figure 2. Illustrates bidirectional drive track, each track before and after a motor drive. There are six intermediate track and the auxiliary drive gear is fixed, an intermediate position assisted by administering long straight lever, the support bar is a gear shape fixation rod.

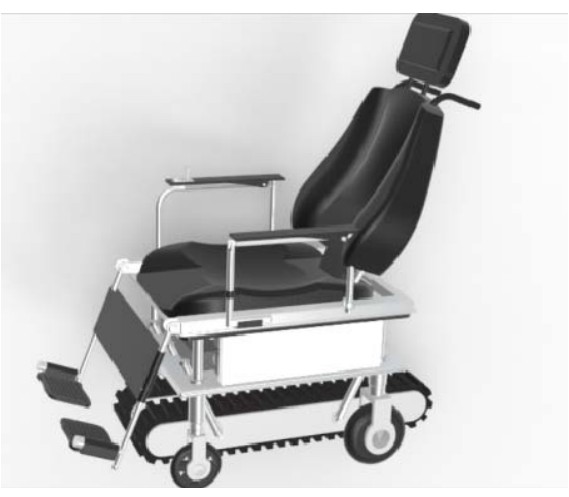

Fig. 2. Structure diagram of moveable track.

\subsection{Drive of auxiliary mechanism}

Since the motion control of the auxiliary mechanism has certain requirements in terms of accuracy, it is advisable to adopt two DC servo brushless motors. First of all, in terms of control, the mechanical characteristics of the DC servo motor can well meet the requirements of the control system. Compared with other control motors, Servo motor control fast response, small inertia can realize fast start and stop, can better ensure the 
safety of users, and generally rated speed can reach 2000 3000 RPM; Strong overload resistance, can withstand three times the rated torque load; The control precision of servo motor control technology is high, which can guarantee the control precision of stair chair. II is undoubtedly necessary to consider the use of this product for people with disabilities. At the same time, the maintenance rate is relatively low. The temperature is low, the efficiency is relatively high, and the life is longer. The driving process of the auxiliary mechanism is shown in Figure 3.

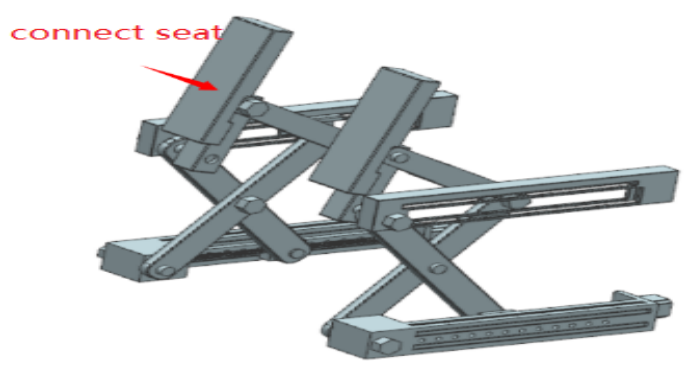

Fig. 3. Drive mode diagram of auxiliary mechanism.

Straight up by the ball screw mechanism with two DC servo brushless motor slide, the center pillar 4, two rails, consisting of a number of screws, this design reduces the space to meet the needs of restructuring, and successfully avoided the conflict with other functional structure, in order to achieve multi-functional integration. At the same time, this also has a mechanism for easy removal and has a simple structure, with flexible.

In the process of realizing the auxiliary standing function, the seat first rises, and then the seat rises and starts to flip at the same time to make the hip change from horizontal to vertical, so that the center of gravity of the person rises first, and then slowly moves forward. Studies have shown that, This design, the law of motion conforms to the dynamic changes of the human body's natural standing process, has good practicability, and forms a triangle after the support is completed, which is relatively stable. Not only that, the material used in this design is simple to manufacture and saves a lot. Reduce the cost and improve the cost performance.

\section{Motion process simulation}

According to the three-dimensional model of the self-service ladder-climbing smart chair established by UG software, in order to optimize its working performance, finite element analysis is carried out. Specific steps are as follows:

(1) The model will be introduced into the overall software UG

(2) The mechanical design of the robot is completed, and the finite element analysis of the structure is carried out

(3) According to the analysis results, modify and correct the parameters of the model itself.

The significance of the simulation model is to shorten the design cycle and reduce the design cost, so the establishment of the simulation model needs a system that can show some characteristics, rather than the whole of the real system. The simulation results show that by applying loads to the motion model and adjusting its structural parameters, the product performance can be effectively improved. Part of the structural finite element analysis results are shown in Figure 4. 


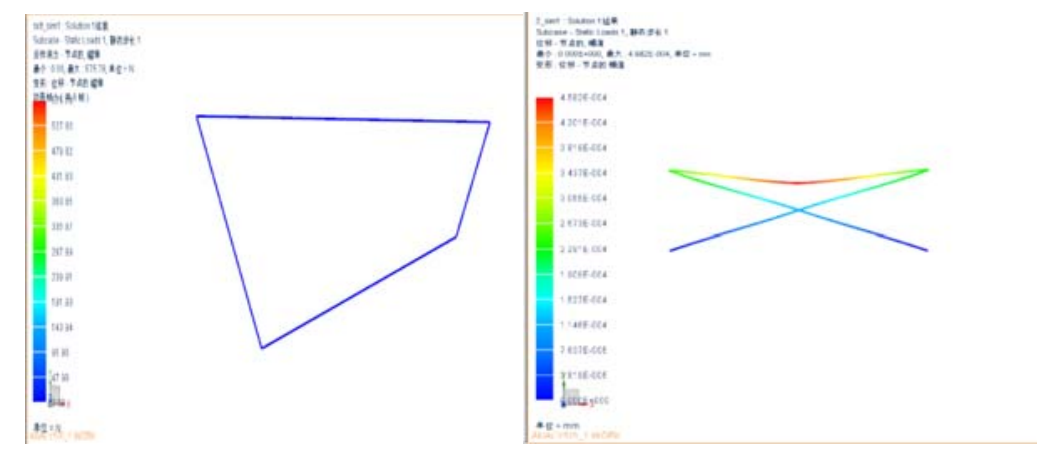

Fig.4. Finite element analysis diagram of partial structure.

\section{Conclusion}

Aiming at the contradiction between supply and demand caused by the current price, quality, function and other problems of the ladder mechanism, a self-help intelligent ladder chair was designed based on ergonomics. The three-dimensional model was established using UG and some finite element analysis, and the model was modeled with reference to actual engineering data. It has gone through the process of modification, prototype construction completion and model rendering.

The cost of the product material is estimated to be about 3500 yuan, and the cost of each wheelchair will increase to about 4800 yuan by increasing the processing fee, assembly fee, management fee, service fee, finance fee, etc. The estimated sales in the first year will be 5500 yuan, and the selling price of each wheelchair will be 7800 yuan after independent operation, that is, the annual sales growth rate will be $37.5 \%$ in 2 to 3 years. The annual sales growth rate in $4-5$ years is $20.8 \%$. The simulation results show that the system structure can effectively improve the comfort of the ladder chair, meet the varied needs of specific user groups and help improve their quality of life in society, as well as create a huge market value

\section{Acknowledgments}

This work was financially supported by Guangdong Province Universities Key Field Fund (2020ZDZX2032) and National Undergraduate Entrepreneurship Practice Project (202013684009S).

\section{References}

1. Liu Haixia's "Thirteenth Five-Year Plan for Healthy Aging" is issued and my country will build a health service system for the elderly[J]. Chinese Medical Information Guide, 2017 (6): 7-7

2. Liu Dongfang. Analysis of the interface design of the elderly website[J]. China Water Transport. 2007. (9): 56-60

3. Lei Hongchun, Zhao Tong, Yang Baoyuan, et al.Design of a convenient wheelchair up and down stairs[J]. Electronic World, 2019(20):162-163.

4. Zhang Tie, Cai Chao. Research on Constant Force Control of Robot Lapping System on Floating Platform [J].Machinery Design \& Manufacture, 2019(6): 150-156. 